\title{
Herramienta informática para calcular las láminas óptimas de agua a aplicar en el riego deficitario de cultivos agrícolas
}

\section{An Informatics tool to calculate the optimum level of water to be applied in deficit irrigation of agricultural crops}

\author{
Jorge Pérez de Corcho ${ }^{1}$, Antonina Capra ${ }^{2}$ \\ ${ }^{1}$ Universidad Central del Ecuador. Facultad de Ciencias Agrícolas. Jerónimo Leiton y \\ Av. La Gasca s/n. Ciudadela Universitaria. 170521 Quito, Ecuador \\ ${ }^{2}$ Dipartimento di Agraria, Università Mediterranea di Reggio Calabria. Salita Melissari - 89124 Reggio Calabria, Italia
}

\begin{abstract}
Resumen
El sector de la agricultura es reconocido como el mayor usuario de agua en el mundo. Un recurso natural cada vez más escaso y contaminado, que además no se utiliza eficientemente. Esto motiva la urgente necesidad de mejorar la eficiencia del uso del agua y el rendimiento de los cultivos bajo riego. Ante esta situación el riego deficitario, o sea, el suministro de agua a los cultivos en cantidad inferior a los niveles de evapotranspiración que se corresponde con la máxima producción, es una solución que potencialmente puede contribuir a un uso más racional del agua, mejorando la eficiencia y competitividad de la producción agrícola a través de la reducción de los costos fijos y de gestión. En el presente trabajo se muestra el uso de MathCad, un programa matemático versátil, de uso general, que tiene múltiples aplicaciones en la solución de problemas de ingeniería, a la determinación de los niveles óptimos de riego en correspondencia con los fundamentos teóricos del riego deficitario. Los resultados demuestran que MathCad es una herramienta adecuada para resolver los problemas de cálculo de los niveles óptimos de riego mencionados.
\end{abstract}

Palabras clave: derivadas, evapotranspiración, eficiencia del riego, optimización, MathCad.

\begin{abstract}
The agriculture sector is the largest user of water in the world. Water is a scarce and polluted natural resource, and a general perception that agricultural water use is often wasteful is widespread. For these reasons, there is an urgent need to use water resources efficiently. Deficit irrigation, the application of irrigation below the full crop evapotranspiration, is potentially able to improve efficiency and maximize profits through a reduction in capital and operating costs. The application of deficit irrigation requires the determination of some optimal irrigation levels which provide maximum profit and/or food production with a limited availability of resources (e.g., land or water). The paper discusses the application of MathCad, a versatile mathematical program commonly used in the solution of engineering problems, to the determination of these optimal irrigation levels. The results showed that MathCad is a tool precise and rapid to solve the problems of calculating these levels.
\end{abstract}

Keywords: derivative, evapotranspiration, irrigation efficiency, optimization, MathCad. 


\section{Introducción}

En regiones con déficit de precipitaciones, el riego es el factor más relevante para obtener rendimientos altos y estables de los cultivos. En estas áreas, la agricultura de regadío es el mayor usuario del agua requiriendo cerca del $70 \%$ del total del agua utilizada (FAO, 2003). Además, se ha difundido la percepción general de que el uso agrícola del agua es poco eficiente y que tiene menor valor que otros usos (Postel, 2000; Jury \& Vaux, 2005). Por estos motivos hay una necesidad urgente de mejorar la eficiencia y la productividad de los cultivos bajo riego (Pereira, 2002). El riego deficitario (RD) es una solución que potencialmente puede mejorar la eficiencia y maximizar la ganancia a través de la reducción de los costos del riego (Capra \& Consoli, 2011).

El riego deficitario surge como un concepto económico en 1970 (James \& Lee, 1971). Las primeras investigaciones sobre RD se publicaron en los primeros años de los 80 (English \& Nuss, 1982; Hargreaves \& Samani, 1984), pero la mayoría de la literatura sobre RD se refiere a las últimas tres décadas (Capra, Consoli \& Scicolone, 2008a).

English (1990), quien desarrolló primero la teoría, definió el RD como "la subirrigación deliberada y sistemática de los cultivos", y desarrolló un método analítico para estimar el máximo nivel de utilidades del uso del agua. Los aspectos económicos del RD están incluidos implícitamente por el autor en la definición dada al considerar que en ciertas circunstancias, el máximo ingreso neto alcanzable en un campo regado puede ser obtenido con RD (English, 1990; English \& Raja, 1996).

Lecler (1998) hace más explícita la definición de English: RD es una estrategia de optimización donde los ingresos netos son maximizados por la reducción de la cantidad de agua aplicada en el riego del cultivo a un nivel que produce alguna reducción del rendimiento causado por stress hídrico. En general, RD es cualquier técnica que prevé suministrar al cultivo cantidades de agua inferiores a aquellas que corresponden con la evapotranspiración que asegura la máxima producción $\left(\mathrm{ET}_{\mathrm{c}}\right)$. El déficit de agua de riego resulta en una baja disponibilidad de agua para las plantas (la cual puede afectar el rendimiento, la calidad de los frutos y las características de la producción) y el cierre parcial de los estomas, lo cual reduce la evapotranspiración ET (Fereres \& Soriano, 2007).
Debemos distinguir al menos tres tipos de $R D$ (Capra et al., 2008a): riego deficitario propiamente dicho ("deficit irrigation" según English \& Nuss, 1982); "regulated deficit irrigation" $(R D I)$ que consiste en someter el cultivo a períodos de stress hídrico en determinadas fases del ciclo cultural que no producen reducciones significativas de la producción; y "partial root drying" ( $P R D)$, que consiste en suministrar un porcentaje de $E T_{c}$ alternando el lado irrigado de la hilera, de manera que cada vez un solo lado de las raíces está en contacto con el terreno húmedo mientras el otro sufre un stress.

El $R D$ propiamente dicho responde la pregunta “cuánto sub-irrigar", el $R D I$ responde a "cuándo aplicar el déficit", el $P R D$ responde a "cómo aplicar el déficit". Las investigaciones sobre RDI pueden también ser vistas como investigaciones sobre las respuestas de los cultivos al agua o sobre los valores óptimos de los coeficientes culturales $K_{c}$ en las diversas fases del ciclo cultural. Ellas constituyen un paso fundamental, pero no el único, para el uso de estrategias de $R D$.

Los factores clave importantes para comprender los beneficios potenciales de la técnica son cuatro (English, 1990; English \& Raja, 1996; Lecler, 1998):

a) la eficiencia del agua de riego disminuye cuando la cantidad de agua se incrementa;

b) la aplicación de agua de riego es costosa;

c) el agua ahorrada por reducción de la irrigación puede ser utilizada para extender el área bajo riego (costo de oportunidad del agua);

d) la determinación de una estrategia ópti ma de riego depende de la escasez de tierra o de si el agua es un factor limitante de la producción.

Generalmente, el $R D$ incrementa la eficiencia del uso del agua por diversas razones (Hsiao, Steduto \& Fereres, 2007; Sepaskhah \& Gharaman, 2004): la eficiencia de la aplicación ( $E_{\text {appl }}=$ agua acumulada a nivel radical/agua suministrada) se incrementa cuando el agua aplicada es menor que la $E T_{c}$, porque la mayoría o toda el agua aplicada permanece en la zona de las raíces (disminuyen las pérdidas de agua por escorrentía y por percolación profunda); la efi- 
ciencia del consumo ( $E_{E T}=$ agua evapotranspirada/ agua acumulada a nivel radical) puede incrementarse porque los cultivos son forzados a extraer grandes niveles de agua del suelo; la eficiencia de la producción ( $E_{y l d}=$ biomasa utilizada/producto cosechado) puede aumentar debido a un crecimiento vegetativo excesivo en algunas especies de cultivos (algodón y uva para vino, por ejemplo) durante el riego completo.

English (1990) y English \& Raja (1996) han identificado diferentes niveles óptimos de erogación de agua que garantizan el máximo rendimiento neto en condiciones de recursos limitados (superficie a irrigar o volumen hídrico) y han desarrollado ecuaciones explícitas para su cálculo. El método analítico desarrollado por estos autores posee cierta complejidad en sus cálculos, a lo cual se unen las dificultades relacionadas con su comprensión, lo cual puede constituir una barrera a su aplicación.

En la práctica profesional es frecuente que los ingenieros se encuentren ante la necesidad de realizar cálculos matemáticos aplicados a la solución de problemas de diferente tipo, para lo cual se cuenta con diversidad de métodos y medios. En este contexto, el uso de programas informáticos permite la realización de cálculos en menor tiempo y con mayor precisión. Existe gran diversidad de programas de cálculos, entre los cuales se encuentra MathCad, el cual goza de gran difusión y popularidad por su facilidad de uso, posibilitando además la demostración de conceptos en un entorno interactivo que permite la modificación de parámetros para valorar los cambios que se producen en los resultados obtenidos.

Por sus características y potencialidades, MathCad es un programa de utilidad para la realización de cálculos de ingeniería, que también puede aplicarse a la determinación de los valores óptimos de láminas de riego, contribuyendo con esto al ahorro del preciado líquido, y a su vez al ahorro de energía y a la competitividad de la producción agrícola.

El propósito del presente trabajo es mostrar el uso del programa MathCad en la determinación de las láminas óptimas de agua a aplicar en el riego deficitario de cultivos agrícolas, facilitando la toma de decisiones a partir de los resultados obtenidos en los cálculos y gráficos realizados. Para desarrollar este ejemplo se toman los resultados de una experimentación realizada en una hacienda agrícola en la provincia de Catania (Italia) en un cultivo de lechuga (Capra, Consoli, Russo \& Scicolone, 2008b).

\section{Materiales y métodos}

La metodología seguida para efectuar los análisis es la propuesta por English (1990) y por English \& Raja (1996), la cual se incluye a continuación para mayor claridad.

La relación entre agua de riego aplicada $(w)$ y rendimiento del cultivo $(y)$ generalmente puede ser expresada por una ecuación cuadrática

$$
y(w)=a_{1}+b_{1} w+c_{1} w^{2}
$$

Los ingresos estimados para el riego $R(w)$, se obtienen multiplicando la función de producción $y(w)$ por una constante (precio del cultivo, $P_{c}$ ), y pueden presentar la misma forma de la función de producción:

$$
R(w)=P_{C} \cdot y(w)
$$

Una posible función relacionada con los costos totales de producción (c) para el agua de riego aplicada es:

$$
c(w)=a_{2}+b_{2} w
$$

El intercepto $\left(a_{2}\right)$ está asociado con los costos fijos: costos de capital, impuestos, seguro, costos fijos de labranza, plantación, uso de productos químicos y cosecha. La pendiente de la línea $\left(b_{2}\right)$ representa los costos marginales variables de producción que incluye: costos variables del riego (costos de agua, bombeo, trabajo y mantenimiento) y otros costos basados en la variación del rendimiento con el uso del agua (costos asociados con la fertilización, cuando los agricultores la adoptan para anticipar las cosechas, costos de cosecha, etc.). English \& Raja (1996) recomiendan analizar los costos directos del riego y otros costos de producción. El análisis incompleto de los costos puede subestimar la magnitud óptima del déficit y la ganancia potencial relacionada con el ingreso neto.

Cuando el agua ahorrada por la reducción de la cantidad aplicada puede ser usada para el riego de terreno adicional, los productores pueden incrementar sus ingresos netos. Este incremento potencial representa un costo de oportunidad de agua. Por ejemplo, una cantidad de agua de $50 \mathrm{~mm}$ puede ser aplicada a 1 ha, o una cantidad de $25 \mathrm{~mm}$ puede ser aplicada a 2 ha, produciendo un incremento del beneficio total. Cuando la disponibilidad de agua es limitada, el rendimiento económico es maximizado reduciendo la cantidad de agua aplicada, e incrementando las áreas bajo 
riego hasta que el producto entre el beneficio marginal por hectárea y el número de hectáreas irrigadas es igual al beneficio total por hectárea con riego completo.

Si la disponibilidad de tierra es limitada, el asunto principal es cuál cantidad de agua produce la máxima diferencia entre el ingreso bruto y los costos. Ya que el nivel óptimo de riego (cuando la tierra o el agua son limitadas) requiere menos agua que ella para obtener el rendimiento máximo, entonces el sistema puede tener poca capacidad y bajos costos de capital. Al bajo costo corresponde la mayor diferencia entre rendimiento de retorno y costos (retorno neto). Obviamente la baja capacidad del sistema representa una limitación en cierta situación, por ejemplo, cuando algunos cultivos son regados en rotación y solo para alguno de ellos las estrategias de $R D$ deben ser aplicadas.

El nivel óptimo de cantidad de agua será aquel que permita obtener el máximo ingreso neto por unidad de agua o de terreno, dependiendo si el agua o el terreno es el factor limitante y si la maximización de la producción de alimentos o de los beneficios es el objetivo principal de la estrategia.

English (1990) ha identificado los siguientes cinco niveles óptimos de aplicación de agua, que proporcionan beneficio y producción máximos con limitada disponibilidad de recursos (ej. tierra o agua):

a) nivel en el cual el rendimiento del cultivo por unidad de terreno es maximizado, $W_{m}$

b) nivel en el cual el ingreso neto por unidad de terreno es maximizado, $W_{l}$

c) nivel en el cual el ingreso neto por unidad de agua es maximizado, $W_{w}$

d) nivel en el cual el ingreso neto es igual al del riego total cuando el terreno es limitado, $W_{e l}$

e) nivel en el cual el ingreso neto iguala al riego total cuando el agua es limitante, $W_{e w}$

El nivel hídrico que maximiza la producción por unidad de superficie, $W_{m}$, representa el óptimo agronómico; en correspondencia con este punto la utilidad marginal es cero, la aplicación de agua no hace aumentar la producción. En el campo de volúmenes hídricos inferiores a $W_{m}$ la utilidad marginal de la última dosis de agua es en cambio mayor que cero y aumenta en la medida que la cantidad de agua disminuye.

El ingreso neto por unidad de superficie es máximo cuando la cantidad de agua alcanza $W_{l}$, nivel en el cual la pendiente de la línea de los costos iguala la de la tangente de la curva del ingreso. A niveles mayores de $W_{l}$, la línea de los costos tiene una pendiente mayor que la de ingresos, o sea los costos crecen más rápidamente que los ingresos. En el intervalo entre $W_{m}$ y $W_{l}$ el agricultor se beneficia de la reducción de los costos.

Sea la eficiencia que el incremento del ingreso neto crecen al disminuir la cantidad de agua, entonces el ingreso neto por unidad de agua es creciente al disminuir el agua suministrada. Si el agua ahorrada reduciendo los volúmenes suministrados es utilizada para regar otras superficies, el ingreso neto entre ambas aumenta. El ingreso neto producido en las superficies adicionales representa el costo de oportunidad del agua. Si el terreno no es un factor limitante, la estrategia de uso del agua que maximiza el ingreso neto sería aquella de irrigar a un nivel inferior a $W_{p}$ indicado por $W_{w}$.

Otros dos niveles interesantes, a pesar de no ser óptimos, son $W_{e l}$ y $W_{e w}$ puntos que se alcanzan reduciendo adicionalmente la cantidad de agua y en los cuales el ingreso neto (diferencia vertical entre las dos líneas) es igual a aquel de la plena irrigación $W_{m}$. El intervalo entre estos dos puntos y $W_{m}$ es el campo útil del riego deficitario, en el cual el ingreso neto (por unidad de superficie o por unidad de agua) es mayor que para la plena irrigación.

English (1990) desarrolló un método analítico para estimar los cinco niveles de utilidades del uso del agua $W_{m}, W_{l}, W_{w}, W_{e l}$ y $W_{e w}$ cuando las ecuaciones de rendimiento y de costo son, respectivamente, de forma cuadrática y lineal (ec. 4 hasta ec. 10):

$$
\begin{aligned}
& W_{m}=-\frac{b_{1}}{2 c_{1}} \\
& W_{l}=-\frac{b_{2}-P_{c} b_{1}}{2 P_{c} c_{1}} \\
& W_{w}=\left(\frac{P_{c} a_{1}-a_{2}}{P_{c}{ }_{1}}\right)^{1 / 2}
\end{aligned}
$$




$$
W_{e l}=\left(\frac{b_{2}-P_{c} b_{1}+Z_{1}}{2 P_{c} c_{1}}\right)
$$

con

$$
\begin{gathered}
Z_{1}=\left[\left(P_{c} b_{1}-b_{2}\right)^{2}-4 P_{c} c_{1} \cdot\left(\frac{P_{c} b_{1}^{2}}{4 c_{1}}-\frac{b_{1} b_{2}}{2 c_{1}}\right)\right]^{1 / 2} \\
W_{e w}=\frac{-Z_{2}+\left[Z_{2}^{2}-4 P_{c} c_{1}\left(P_{c} a_{1}-a_{2}\right)\right]^{1 / 2}}{2 P_{c} c_{1}}
\end{gathered}
$$

con

$$
Z_{2}=\frac{P_{c} b_{1}^{2}-4 a_{2} c_{1}+4 P_{c} a_{1} c_{1}}{2 b_{1}}
$$

Para calcular los valores máximos las funciones estudiadas se aplica la condición necesaria para la existencia de un valor extremo relativo de dicha función cuyo punto crítico se obtiene para el valor en el cual la derivada en este punto se reduce a cero (Riquenes y García, 2008). Para el caso que nos ocupa se determinará el valor de la lámina de agua $w$ para el cual la primera derivada de la función de se hace cero, en correspondencia con los criterios de optimización fundamentados por English (1990), es decir, se determina el valor de $w$ para el cual $\frac{d}{d w} f(w)=0$.

MathCad dispone de varios métodos para calcular esto. Uno de ellos, el más sencillo, es el método de Newton, en el cual se basa la función root de MathCad. El formato del comando root es root (expresión,variable), donde la expresión puede ser una función, la derivada de una función, la diferencia entre dos funciones, etc. El comando root devuelve como respuesta el valor de la variable que anula la expresión, el valor de la variable que hace la expresión igual a cero.

Con MathCad se obtienen los valores máximos de las funciones correspondientes utilizando la sintaxis, $\operatorname{root}\left[\frac{d}{d w} f(w), w\right]$ que devuelve el valor de para el cual $\frac{d}{d w} f(w)=0$. Utilizando Mathcad tendríamos: a) nivel en el cual el rendimiento del cultivo por unidad de terreno es maximizado, $W_{m}$

$w_{m}(w):=\operatorname{root}\left(\frac{d}{d w} y(w), w\right)$

b) nivel en el cual el ingreso neto por unidad de terreno es maximizado, $W_{l}$

$\mathrm{p}(\mathrm{w}):=\mathrm{R}(\mathrm{w})-\mathrm{c}(\mathrm{w})$

$w_{1}(w):=\operatorname{root}\left(\frac{d}{d w} p(w), w\right)$

c) nivel en el cual el ingreso neto por unidad de agua es maximizado, $W_{w}$

$\mathfrak{r}(w):=\frac{\mathrm{p}(w)}{w} \quad w_{w}(w):=\operatorname{root}\left(\frac{d}{d w} \mathfrak{r}(w), w\right)$

d) nivel en el cual el ingreso neto es igual al del riego total cuando el terreno es limitado, $W_{e l}$

$\mathrm{w}_{\mathrm{e} 1}(\mathrm{w}):=\operatorname{root}\left(\mathrm{p}\left(\mathrm{w}_{\mathrm{m}}(0)\right)-\mathrm{p}(\mathrm{w}), \mathrm{w}\right)$

(Con este nivel de riego el ingreso neto, es decir, ingreso menos costo, es igual al obtenido para el nivel de riego de máximo rendimiento del cultivo).

e) nivel en el cual el ingreso neto iguala al riego total cuando el agua es limitante, $W_{e w}$

En el programa elaborado se obtiene aplicando la metodología de English (1990).

Para desarrollar este ejemplo de determinación de las láminas óptimas de agua de riego se toman los resultados de una experimentación realizada en una hacienda agrícola en la provincia de Catania, en el curso de la estación de riego del año 2005, en un cultivo de lechuga tipo Batavia roja variedad Emini (ciclo cultural estival $\sim 50$ días hasta la plena maduración), donde se obtuvieron los siguientes resultados para el análisis (Capra, Consoli \& Scicolone, 2008c):

Precio del cultivo (Euro/t):

$P_{C}=470$

Función de producción ( $\mathrm{t} / \mathrm{ha})$ :

$y(w)=-0.00054 \cdot w^{2}+0.2654 \cdot w+25.3639$ 
Ingresos estimados para el riego (Euro/ha):

$$
\begin{aligned}
& R(w)=P_{c} \cdot y(w) \\
& R(w)=-0.2549 \cdot w^{2}+124.7408 \cdot w+11921.033
\end{aligned}
$$

Función de costo económico (Euro/ha):

$c(w)=0.0121 \cdot w^{2}-2.5453 \cdot w+14083$

\section{Resultados y discusión}

Se presenta la comparación de los resultados obtenidos con el método propuesto, aplicando MathCad, con los de English (1990) calculados de la forma tradicional, para los niveles óptimos correspondientes a las ecuaciones de la (4) a la (7), y también se obtiene el valor resultante para las ecuaciones (9) y (10). En las figuras 1 a 5 se reportan los gráficos obtenidos con MathCad.

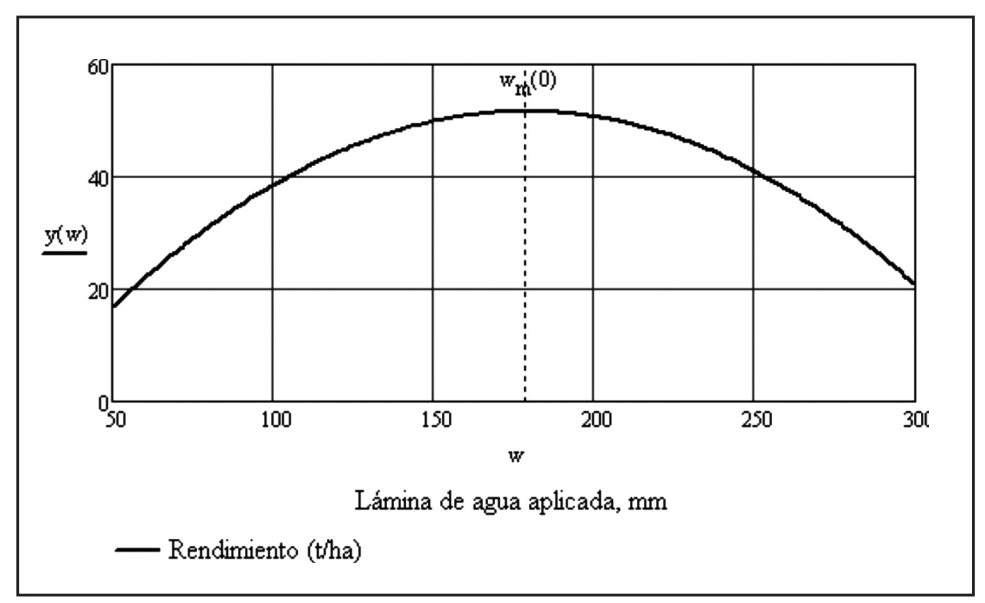

Figura 1. Nivel hídrico que maximiza la producción por unidad de superficie $\left(W_{m}\right)$.

El nivel en el cual el rendimiento del cultivo por unidad de terreno (óptimo agronómico) es maximizado, $W_{m}$, corresponde a $178.9 \mathrm{~mm}$ utilizando los dos métodos.

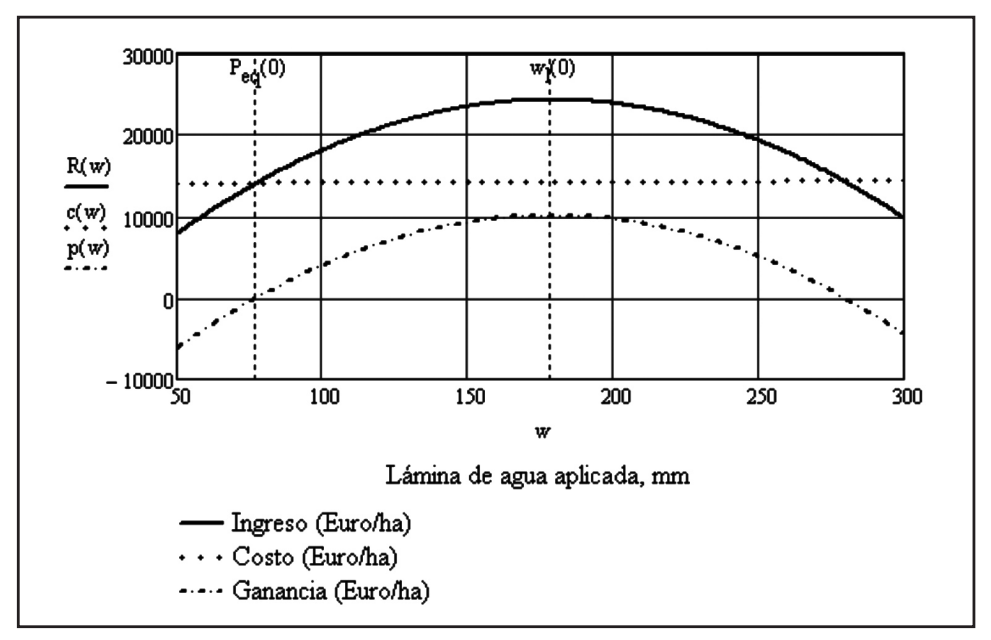

Figura 2. Nivel hídrico que maximiza el ingreso neto por unidad de terreno $\left(W_{l}\right)$.

El nivel en el cual el ingreso neto por unidad de terreno es máximo, $W_{l}$, es igual a $178.29 \mathrm{~mm}$ utilizando tanto MathCad como la ecuación de English. El punto de equilibrio en el cual la ganancia por riego es cero, calculado con MathCad
$\left(P_{e q}(w)=\operatorname{root}(\mathrm{p}(w), w)\right)$, es $77.2 \mathrm{~mm}$. El nivel en el cual el ingreso neto por unidad de agua es maximizado, $W_{w}$, resultó inferior a $W_{l}$ siendo igual a $146.86 \mathrm{~mm}$. También en este caso no se obtuvieron diferencias entre los dos métodos de cálculo. 
Asimismo para el nivel en el cual el ingreso neto es igual al del riego total (sin déficit aplicado) cuando el terreno es limitado, $W_{e l}$, es de 177.67 $\mathrm{mm}$, sin diferencias con ambos métodos.

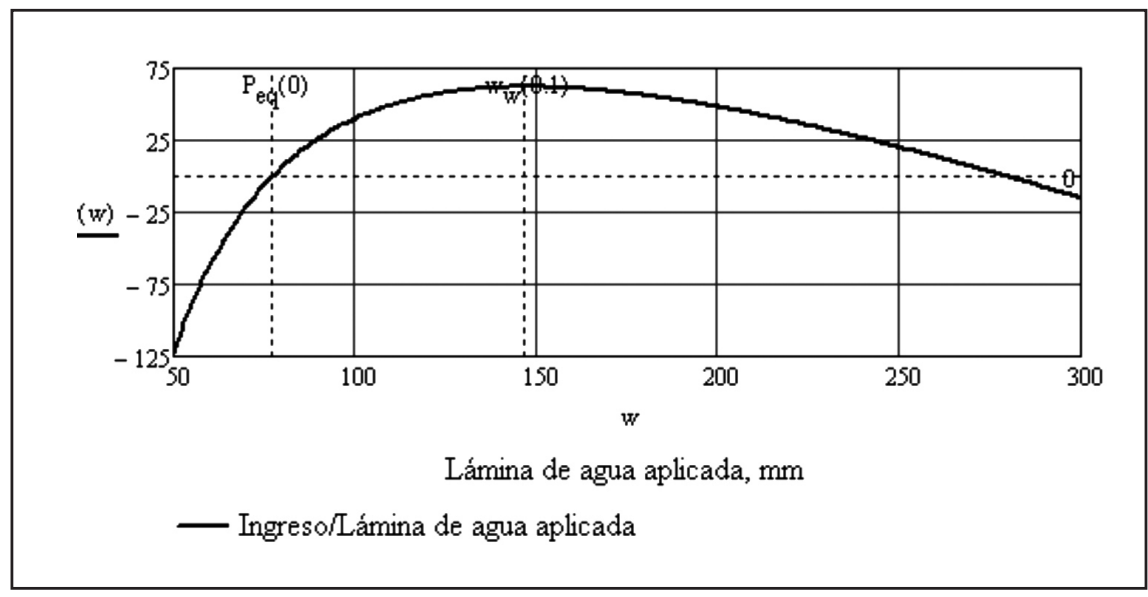

Figura 3. Nivel hídrico que maximiza el ingreso neto por unidad de agua $\left(W_{w}\right)$.

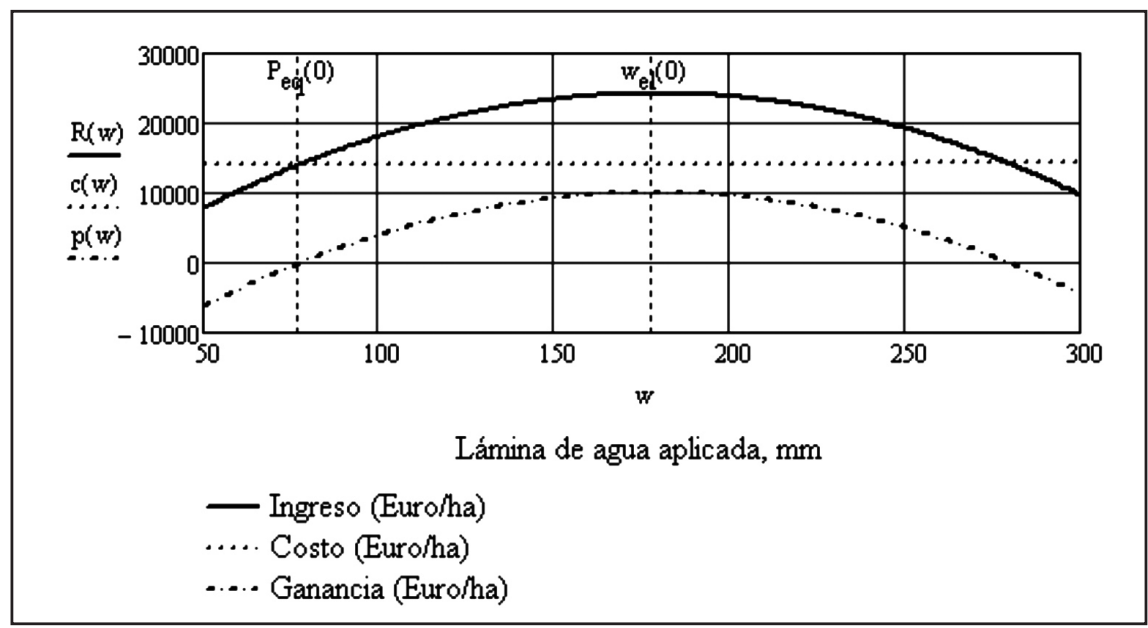

Figura 4. Nivel hídrico en el cual el ingreso neto es igual al del riego total cuando el terreno es limitado $\left(W_{e l}\right)$.

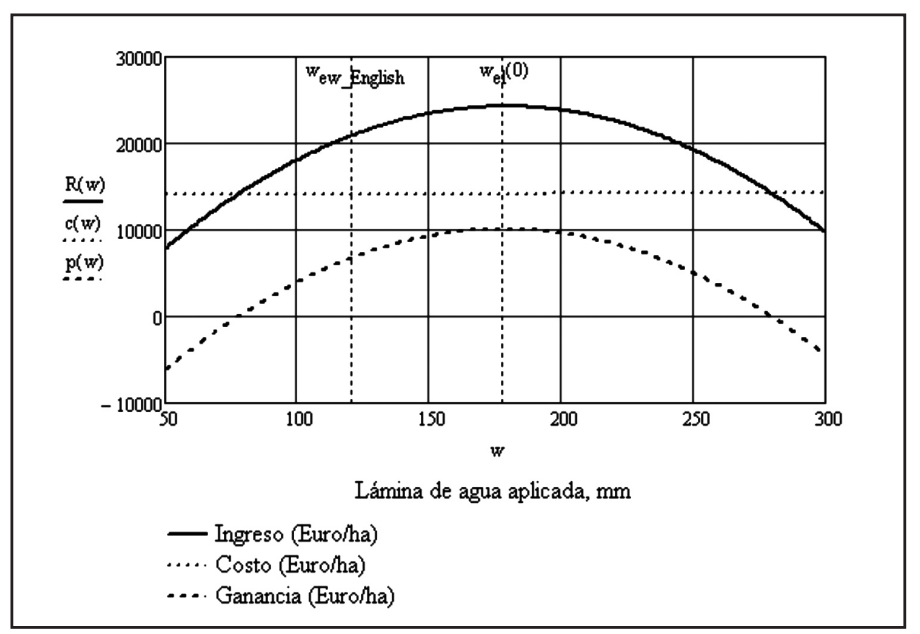

Figura 5. Nivel hídrico en el cual el ingreso neto es igual al del riego total cuando el agua es limitada $\left(W_{e w}\right)$. 
Finalmente, el nivel en el cual el ingreso neto iguala al riego total cuando el agua es limitante, $W_{e w}$ también calculado por ambos métodos, dio como resultado $120.55 \mathrm{~mm}$.

En el caso examinado, el máximo rendimiento de la lechuga (óptimo agronómico, $W_{m}$ ) se alcanzó con aproximadamente $180 \mathrm{~mm}$ de agua aplicada en el riego. Si el terreno es limitado, los niveles de agua aplicada (correspondientes al óptimo económico, $W_{l}$, y al déficit en el cual la ganancia iguala al riego total, $W_{e l}$ ), han sido muy próximos a $W_{m}$. Este resultado es coherente con el hecho de que de la lechuga se utiliza su biomasa. Si el agua es limitada (y esto es possible para aumentar el area bajo riego), el máximo ingreso neto puede ser obtenido resuciendo la aplicación de agua de $W_{m}$ a $W_{w}$. El nivel óptimo de agua debe ser $147 \mathrm{~mm}$, con un ahorro de $33 \mathrm{~mm}\left(330 \mathrm{~m}^{3} / \mathrm{ha}\right)$ cerca del $18 \%$, que podrían ser utilizados para ampliar la superficie irrigada si el interés fuese maximizar el rédito global de una región, en vez de una sola hacienda.

\section{Conclusiones}

Como se ha observado, en el cálculo de los niveles de riego óptimos a suministrar en el riego

\section{Referencias}

Capra, A., Consoli, S. \& Scicolone, B. (2008a). Deficit irrigation: theory and practice. In Alonso, D. and Iglesias, H.J. (Eds), Agricultural Irrigation Research Progress, Nova Science Pub., USA, 53-82.

Capra, A.; Consoli, S.; Russo, A. \& Scicolone, B. (2008b). Integrated agro-economic approach to deficit irrigation on lettuce crops in Sicily (Italy). Journal of Irrigation and Drainage Division (ASCE), 134(4), 437-445.

Capra, A.; Consoli, S. \& Scicolone, B. (2008c). Water management strategies under deficit irrigation. Journal of Agricultural Engineering - Rivista di Ingegneria Agraria, 4, 47-56.

Capra, A. \& Consoli, S. (2011). Economic Analysis of Citrus Orchards under Deficit Irrigation in South Italy. Acta Hort. 922, ISHS 2011, 209-215.

English, M.J. (1990). Deficit irrigation: an analytical framework. Journal of Irrigation and Drainage Engineering, ASCE, 116(3), 399-412. deficitario existe coincidencia en los resultados obtenidos aplicando las ecuaciones propuestas por English o aplicando los métodos de cálculo con el uso de MathCad, lo que permite utilizar uno u otro.

El uso de MathCad facilita la realización de los cálculos y la visualización gráfica de los resultados, lo que contribuye a la toma de decisiones en la producción agrícola. Con el uso del MathCad, además, se reduce la posibilidad de cometer errores y se disminuye el tiempo de cálculo. También cuando varían las condiciones de producción (tipo y precio de cultivo, funciones de producción y de costo, etc.), es posible determinar en forma rápida y precisa los niveles óptimos de las láminas de agua a aplicar en el riego deficitario, y esta posibilidad es particularmente interesante en los casos en los cuales son necesarias numerosas simulaciones.

Además, el procedimiento desarrollado puede ser de utilidad en la docencia universitaria, permitiendo la vinculación de conceptos matemáticos y económicos con la realidad productiva en el sector agrícola.

English, M.J. \& Nuss, G.S. (1982). Designing for deficit irrigation. Journal Irrigation and Drainage Engineering, ASCE, 108(2), 91-106.

English, M.J. (1990). Deficit irrigation: an analytical framework. Journal of Irrigation and Drainage Engineering, ASCE, 116 (3), 399-412.

English, M.J. \& Raja, S.N. (1996). Perspective on deficit irrigation. Agricultural Water Management, $32,1-14$.

FAO (2003). Unlocking the water potential of agriculture. Food and Agriculture Organization of the United Nations.

Fereres, E. \& Soriano, A. (2007). Deficit irrigation for reducing agricultural water use. J. Exp. Bot. 58, 147-159.

Hargreaves, G.H. \& Samani, Z.A. (1984). Economic considerations of deficit irrigation. Journal Irrigation and Drainage Division, ASCE, 110(4), 343-358. 
Hsiao, T.C.; Steduto, P. \& Fereres, E. (2007). A systematic and quantitative approach to improve water use efficiency in agriculture. Irrigation Science, 25, 209-231.

James, L.D. \& Lee, R.R. (1971). Economics of water resources planning. New York, USA, McGraw-Hill, 325.

Jury, W.A. \& Vaux, H. Jr. (2005). The role of science in solving the world's emerging water problems. Proc Natl Acad Sci, 102, 15715-15720.

Lecler, NL. (1998). Integrated methods and models for deficit irrigation planning. In RM Pert; RB Curry (Ed.), Agricultural systems modelling and simulation, Marcel Dekker.
Pereira, L.S. (2002). Irrigation demand management to cope with drought and water scarcity. In Rossi, G., Cancelliere, A., Pereira, L.S., Oweis, T., Shatanawi, M., Zairi, A. (eds). Tools for drought mitigation in Mediterranean regions.

Postel, S. (2000). Entering an era of water scarcity: the challenges ahead. Ecol. Appl., 10, 941-948.

Riquenes Rodríguez, M. \& García Pérez, I. (2008). Resolución de problemas de optimización a través del cálculo diferencial. Ciudad de La Habana. Editorial Universitaria.

Sepaskhah, A.R. \& Gharaman, B. (2004). The effects of irrigation efficiency and uniformity coefficient on relative yield and profit for deficit irrigation. Biosystems Engineering, 87(4), 495-507. 